\title{
Mid-IR Imaging of Orion BN/KL: Modeling of Physical Conditions and Energy Balance
}

\author{
Daniel Gezari ${ }^{1}$, Frank $\operatorname{Varosi}^{2}$, Eli Dwek ${ }^{1}$, William Danchi ${ }^{1}$, \\ Jonathan Tan $^{2}$, and Shin-ichiro Okumura ${ }^{3}$ \\ ${ }^{1}$ Code 667, NASA/GSFC, Greenbelt, MD 20771 USA; email: Daniel.Y.Gezari@nasa.gov \\ ${ }^{2}$ Univ. of Florida-Gainesville, USA; ${ }^{3}$ Okayama Astro. Observatory, Japan
}

We have modeled two mid-infrared imaging photometry data sets to determine the spatial distribution of physical conditions in the BN/KL infrared complex. We observed the BN/KL region using the 10-m Keck I telescope and the LWS in the direct imaging mode, over a $13^{\prime \prime} \times 19^{\prime \prime}$ field (Figure 1, left). We also modeled images obtained with COMICS (Kataza et al.2000) at the 8.2-m SUBARU telescope, over a total field of view is $31^{\prime \prime} \times 41^{\prime \prime}$ (Figure 1, right), in a total of nine bands: $7.8,8.8,9.7,10.5,11.7,12.4,18.5,20.8$ and $24.8 \mu \mathrm{m}$ with $\sim 1 \mu \mathrm{m}$ bandwidth interference filters.
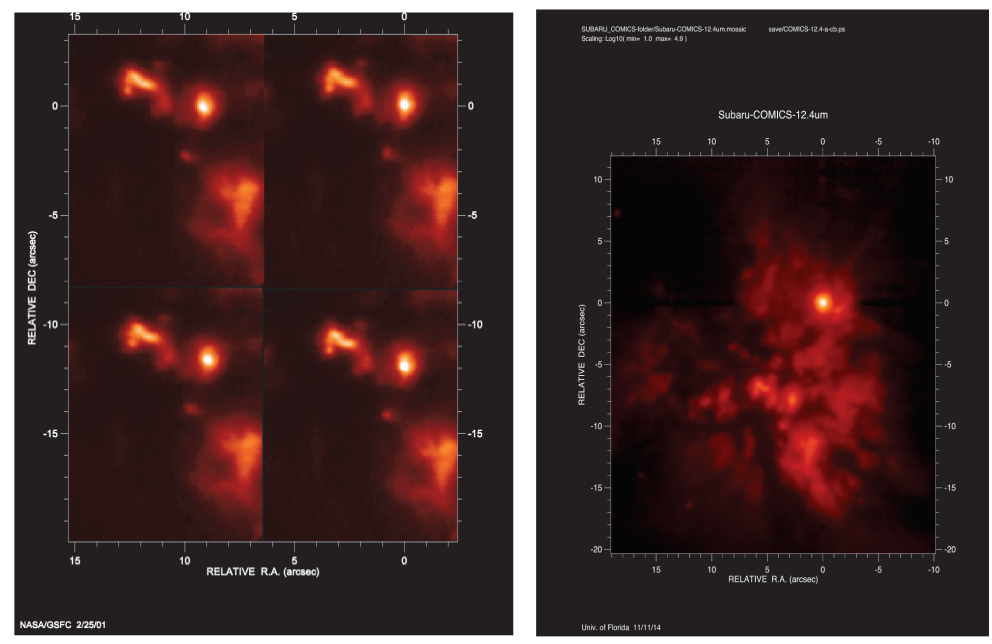

Figure 1. (Left): Keck single-frame, short exposure $12.4 \mu \mathrm{m}$ "speckle" images of the BN/KL core cluster capturing new $\sim 0.3^{\prime \prime}$ seeing-limited source detail. (Right): COMICS $12.5 \mu \mathrm{m}$ wide field image (Okumura et al.2011).

We applied the new two-temperature version (Varosi 2015) of our planar, line-of-sight (LOS), radiative transfer dust model (Dwek and Varosi 1998) to each mid-infrared nine-image stack. Attempts to fit both mid-infrared and near infrared data with a single dust temperature did generally do not yield satisfactory fits. The two-temperature LOS model is a better approximation of the true source morphology. In the model, the dust is actually arranged as three components along the LOS, i.e., 1) the warm emitting dust at the source, 2) the cool emitting dust near the source, and 3) a third component where a fraction of the cool emitting dust is a foreground absorbing layer along the LOS.

The Keck modeling results show that the hot dust temperature distribution peaks along the southeast edge of IRc2, and east of "n", very close to the position of radio source I. (The model cannot compute results at "I" because of insufficient S/N of mid-IR emission at that position). The warm dust temperature distribution peaks at the positions of the prominent central cluster mid-IR compact sources IRc2, IRc7 and IRc4/KL. Note that the warm dust contribution is minimal near the position of radio source "I" (south of IRc2). The combined results suggest that the hottest dust is associated with radio source "I". The luminosity distribution of hot dust 
peaks at the southern extent of IRc2A (closest to "I"). Note that the compact ionizing source " $\mathrm{n}$ " is a distinct peak in the hot dust luminosity. In contrast, the luminosity distribution of warm dust peaks at IRc2A and IRc2B, IRc7 and IRc4/KL, but not at "n". These results suggest that the hot dust and warm dust are physically separate cloud components. The modeled mid-IR luminosity results for the $\mathrm{BN} / \mathrm{KL}$ core cluster sources are: $\mathrm{BN}=4300 L_{\odot}, \mathrm{KL}=2500 L_{\odot}$, IRc2 + Radio I $=3700 L_{\odot}$, and total for the BN/KL core $\sim 20,000 L_{\odot}$. The opacity of warm emitting dust at $12.4 \mu \mathrm{m}$ is relatively smooth in the central cluster. The model results suggest that temperature and extinction effects, rather than density knots, determine the observed midIR emission distribution of BN/KL.

The COMICS modeling results show that opacity of hot dust $(T \sim 1000 \mathrm{~K})$ is similar among all the prominent mid-IR sources in BN/KL, implying that the dust densities are comparable among the emitting dust clouds in the complex. This suggests that luminosity differences are due to variations in local dust temperature, not density. However, the opacity of warm dust is minimum in the quadrant south-east of BN, near the bright sources IRc2, IRc7, and the position of radio source "I". This suggests that warm $(T \sim 300 \mathrm{~K})$ dust is not the dominant emission component. The opacity of cold $(T<100 \mathrm{~K})$ dust is a measure of LOS extinction, since cold dust does not emit significantly at mid-IR wavelengths. It is highest in the quadrant south-east of $\mathrm{BN}$, peaking at the bright source IRc2 and the position of radio source "I", and extending southwest to KL/IRc4. This implies that the cold dust is circumstellar, or local to the emitting compact sources, which would account for the high derived luminosities of IRc2 and radio source "I".

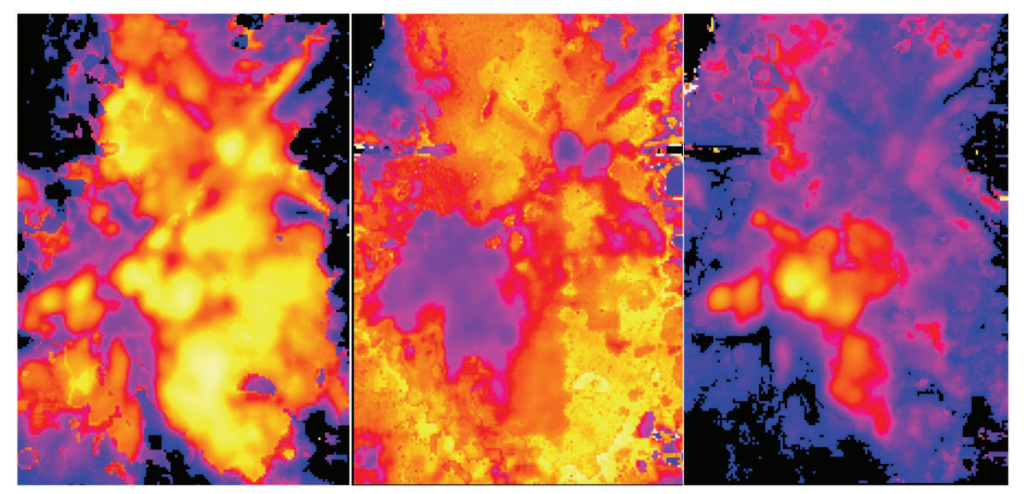

Figure 2. Modeled hot, warm and cool dust opacity from COMICS image data. Left: The opacity of hot dust $(T \sim 1000 \mathrm{~K})$ is similar among all the mid-IR sources in BN/KL. Center: The opacity of warm dust is minimum near IRc2, IRc7, and radio source "I". Right: Cold dust $(T<100 \mathrm{~K})$ opacity, a measure of LOS extinction, is highest at IRc2 and radio source "I".

\section{References}

Kataza, H., Okamoto Y., Takubo, S., Onaka, T., Sako, S., Nakamura, K., Miyata, T., Yamashita, T. 2000, Proc. SPIE, 4008, 1144.

Okumura, S., Yamashita, T., Sako, S., Miyata, T., Honda, M., Kataza, H., Okamoto, Y. 2011 PASJ, 63 (4), 823-834.

Dwek, E. and Varosi, F. 1998, NASA/Goddard - Technical Report.

Varosi, F., 2015, private communication. 